\title{
Bi-doped ceria as a highly efficient catalyst for soot combustion: improved mobility of lattice oxygen in $\mathrm{Ce}_{\mathrm{x}} \mathrm{Bi}_{1-\mathrm{x}} \mathrm{O}_{\mathrm{y}}$ catalysts
}

Bing Cui ${ }^{a}$, Yan Li ${ }^{\text {a }}$, Shuirong $\mathrm{Li}^{\mathrm{a}^{*}}$, Yongkang Xia ${ }^{\mathrm{b}}$, Zhifeng Zheng ${ }^{\mathrm{a}}$, Yun-Quan Liu ${ }^{\mathrm{a}^{*}}$

a College of Energy, Collaborative Innovation Center of Chemistry for Energy Materials (iChEM), Xiamen University, Xiamen, 361102, PR China

b National Engineering Laboratory for Green Chemical Production of Alcohols-Ethers-Esters, Department of Chemical and Biochemical Engineering, College of Chemistry and Chemical Engineering, Xiamen University, Xiamen, 361102, P.R. China

*Author to whom all correspondence should be addressed. E-mail address: sli@xmu.edu.cn, yq liu@xmu.edu.cn, Fax: (+86)-592-2188053; Tel:(+86)-5925952780 
Table S1

Comparison of catalytic activities of Bi-containing catalysts from this work with those from literature.

\begin{tabular}{|c|c|c|c|c|c|c|c|c|c|}
\hline sample & reaction gas & $\begin{array}{l}\text { contact } \\
\text { mode }\end{array}$ & $\begin{array}{c}\text { catalyst } \\
\text { : soot }\end{array}$ & $\begin{array}{c}\text { gas } \\
\text { flow } \\
(\mathrm{ml} / \mathrm{min} \\
)\end{array}$ & $\begin{array}{c}\text { heatin } \\
\text { g rate } \\
\left(^{\circ}\right. \\
\mathrm{C} / \mathrm{min} \\
)\end{array}$ & $\begin{array}{c}T_{50} \text { or } \\
T_{\mathrm{m}} \\
\left({ }^{\circ} \mathrm{C}\right)\end{array}$ & stability & aged & ref \\
\hline $\begin{array}{c}\mathrm{Bi}_{2} \mathrm{O}_{3} \\
\mathrm{Bi}_{0.2} \mathrm{Fe}_{1.8}\end{array}$ & $\begin{array}{c}10 \% \mathrm{O}_{2} \text { in } \\
\mathrm{Ar}+10 \mathrm{vol} \% \\
\mathrm{H}_{2} \mathrm{O}\end{array}$ & tight & $10: 1$ & 50 & 5 & $\begin{array}{l}443 \\
395\end{array}$ & & & [1] \\
\hline $\mathrm{Bi}_{0.2} \mathrm{Co}$ & $8 \% \mathrm{O}_{2}$ in $\mathrm{Ar}$ & $\begin{array}{l}\text { tight } \\
\text { loose }\end{array}$ & $9: 1$ & 50 & 10 & $\begin{array}{l}332 \\
475\end{array}$ & $\Delta 5$ (14cycles $)$ & & [2] \\
\hline $\begin{array}{c}\mathrm{Ce}_{0.86} \mathrm{Zr}_{0.14} \mathrm{O}_{2.0} \\
\mathrm{Ce}_{0.68} \mathrm{Zr}_{0.18} \mathrm{Bi}_{0.14} \mathrm{O}_{1.93}\end{array}$ & Synthetic air & & $50: 1$ & 20 & 5 & $\begin{array}{l}447 \\
383\end{array}$ & & $\begin{array}{l}420 \\
400\end{array}$ & [3] \\
\hline $\mathrm{Bi}_{2} \mathrm{Ce}_{8}$ & $\begin{array}{l}10 \mathrm{vol} \% \mathrm{O}_{2} \\
\quad / \mathrm{Ar}\end{array}$ & $\begin{array}{l}\text { tight } \\
\text { loose }\end{array}$ & $9: 1$ & 200 & 5 & $\begin{array}{l}358 \\
465\end{array}$ & & & [4] \\
\hline $\mathrm{Ce}_{0.8} \mathrm{Bi}_{0.2} \mathrm{O}_{\mathrm{x}}$ & Synthetic air & $\begin{array}{l}\text { tight } \\
\text { tight }\end{array}$ & $4: 1$ & 25 & 5 & $\begin{array}{l}444 \\
324\end{array}$ & & & {$[5]$} \\
\hline $\mathrm{Ce} 0.5 \mathrm{Bi} 0.5 \mathrm{O}_{\mathrm{y}}$ & $5 \% \mathrm{O}_{2}$ in $\mathrm{Ar}$ & loose & $10: 1$ & 50 & 5 & 412 & $\begin{array}{c}\Delta 21 \\
(3 \text { cycles })\end{array}$ & & $\begin{array}{l}\text { This } \\
\text { work }\end{array}$ \\
\hline
\end{tabular}

\section{References:}

[1] Xie, Y.; Zhang, C.; Wang, D.; Lu, J.; Wang, Y.; Wang, J.; Zhang, L.; Zhang, R. Catalytic performance of a $\mathrm{Bi}_{2} \mathrm{O}_{3}-\mathrm{Fe}_{2} \mathrm{O}_{3}$ system in soot combustion. New Journal of Chemistry. 2019, $43,38,15368-15374$.

[2] Shang, Z.; Sun, M.; Chang, S.; Che, X.; Cao, X.; Wang, L.; Guo, Y.; Zhan, W.; Guo, Y.; Lu, G. Activity and stability of $\mathrm{Co}_{3} \mathrm{O}_{4}$-based catalysts for soot oxidation: The enhanced effect of $\mathrm{Bi}_{2} \mathrm{O}_{3}$ on activation and transfer of oxygen. Appl. Catal. B: Environ. 2017, 209, 33-44.

[3] Masui, T.; Minami, K.; Koyabu, K.; Imanaka, N. Synthesis and characterization of new promoters based on $\mathrm{CeO}_{2}-\mathrm{ZrO}_{2}-\mathrm{Bi}_{2} \mathrm{O}_{3}$ for automotive exhaust catalysts. Catal. Today. 2006, $117,1-3,187-192$

[4] Yang, Y.; Xing, L.; Ren, W.; Zhao, D.; Jian, S.; Cao, C.; Wang, C.; Tian, Y.; Yu, X.; Li, X. Oxygen activation through $\beta-\mathrm{Bi}_{2} \mathrm{O}_{3}$ and ultrafine $\mathrm{CeO}_{2}$ interactions to promote catalytic soot combustion. Ind. Eng. Chem. Res. 2019, 58, 48, 22006-22014.

[5] Hebert, S. C.; Stowe, K. Synthesis and characterization of bismuth-cerium oxides for the 
catalytic oxidation of diesel soot. Materials. 2020, 13, 6, 1369-1383. 


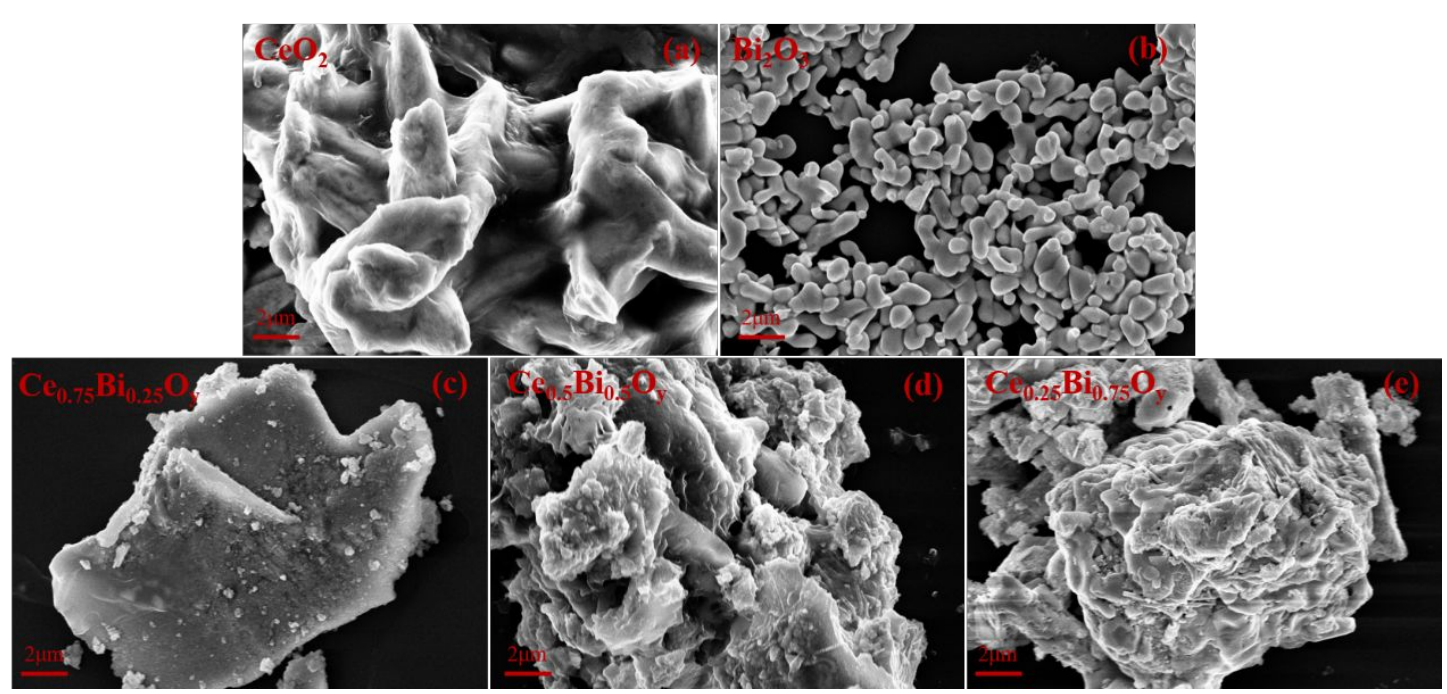

Fig. S1. SEM images of the morphology of $\mathrm{CeO}_{2}(\mathrm{a}), \mathrm{Bi}_{2} \mathrm{O}_{3}(\mathrm{~b}), \mathrm{Ce}_{0.75} \mathrm{Bi}_{0.25} \mathrm{O}_{\mathrm{y}}(\mathrm{c}), \mathrm{Ce}_{0.5} \mathrm{Bi}_{0.5} \mathrm{O}_{\mathrm{y}}$ (d) and $\mathrm{Ce}_{0.25} \mathrm{Bi}_{0.75} \mathrm{O}_{\mathrm{y}}$ (e). 


\section{Table S2}

Textural properties of the catalysts.

\begin{tabular}{cccccc}
\hline sample & $\begin{array}{c}\text { Specific surface } \\
\text { area } S_{\mathrm{BET}}\left(\mathrm{m}^{2} \mathrm{~g}^{-1}\right)^{\mathrm{a}}\end{array}$ & $\begin{array}{c}\text { Pore volume } \\
V_{\mathrm{p}}\left(\mathrm{cm}^{3} \mathrm{~g}^{-1}\right)^{\mathrm{a}}\end{array}$ & $\begin{array}{c}\text { Pore diameter } \\
D_{\mathrm{p}}(\mathrm{nm})^{\mathrm{a}}\end{array}$ & $\begin{array}{c}\text { Crystallite size } \\
D(\mathrm{~nm})^{\mathrm{b}}\end{array}$ & $\begin{array}{c}\text { Lattice constant } \\
a(\AA)^{\mathrm{c}}\end{array}$ \\
\hline $\mathrm{Ce}_{0.5} \mathrm{Bi}_{0.5} \mathrm{O}_{\mathrm{y}}$ & 43 & 0.21 & 6.0 & 6.5 & 5.4793 \\
$\mathrm{Ce}_{0.5} \mathrm{Bi}_{0.5} \mathrm{O}_{\mathrm{y}}{ }^{3 r d}$ & 38 & 0.20 & 5.1 & 7.4 & 5.4665 \\
\hline
\end{tabular}

${ }^{a}$ The BET surface areas, pore volume and pore size for all samples derived from $\mathrm{N}_{2}$ physisorption analysis.

$\mathrm{b}$ The crystallite size value based on the full width at half maximum (FWHM) of most intense (111) plane was calculated by using the Scherrer equation.

${ }^{\mathrm{c}}$ The lattice constant and $d$-spacing of (111) plane was calculated through a Rietveld refinement. 


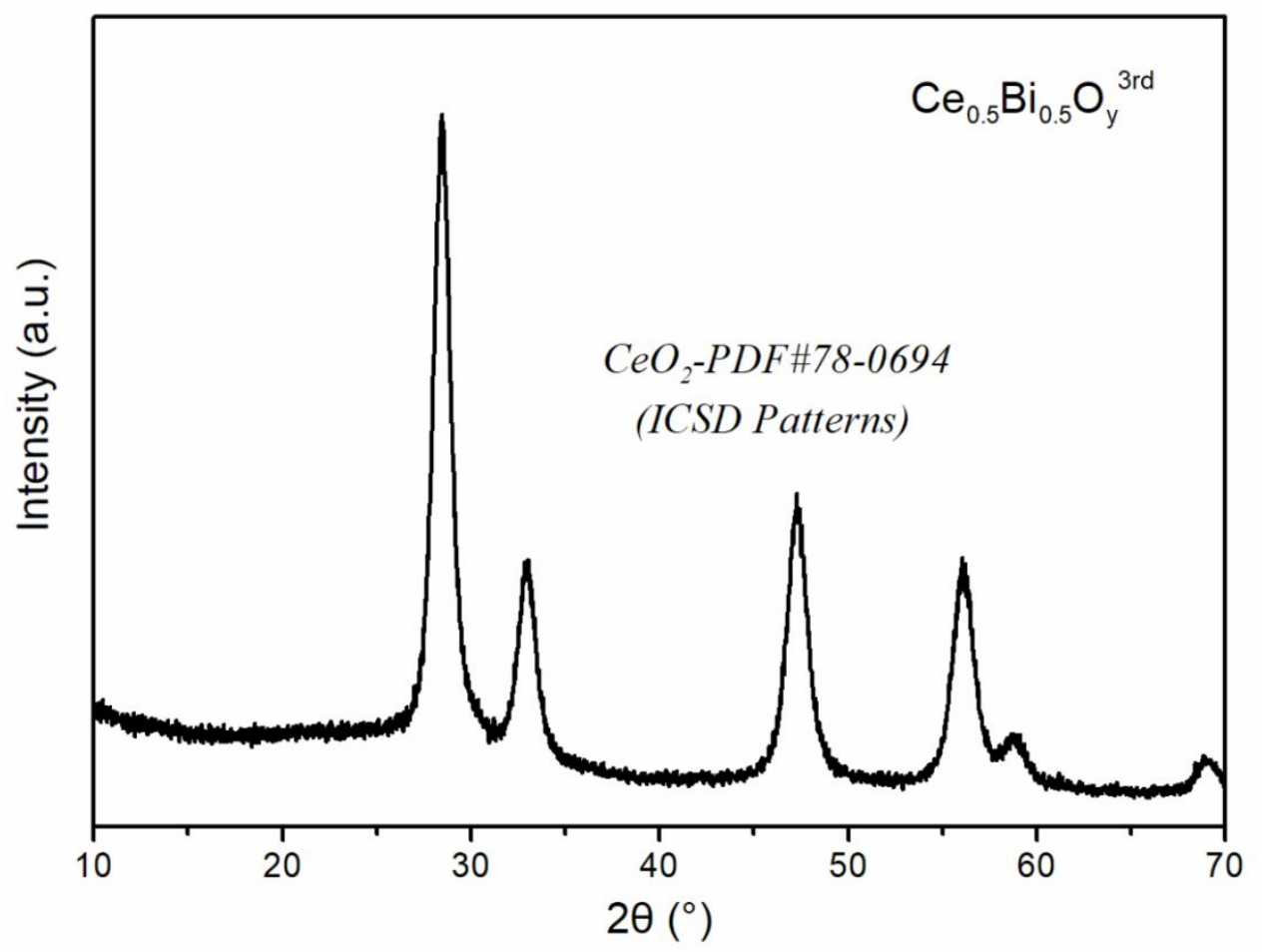

Fig. S2. X-ray diffraction patterns of $\mathrm{Ce}_{0.5} \mathrm{Bi}_{0.5} \mathrm{O}_{\mathrm{y}}$ after the three cycles of testing at loose contact mode. 


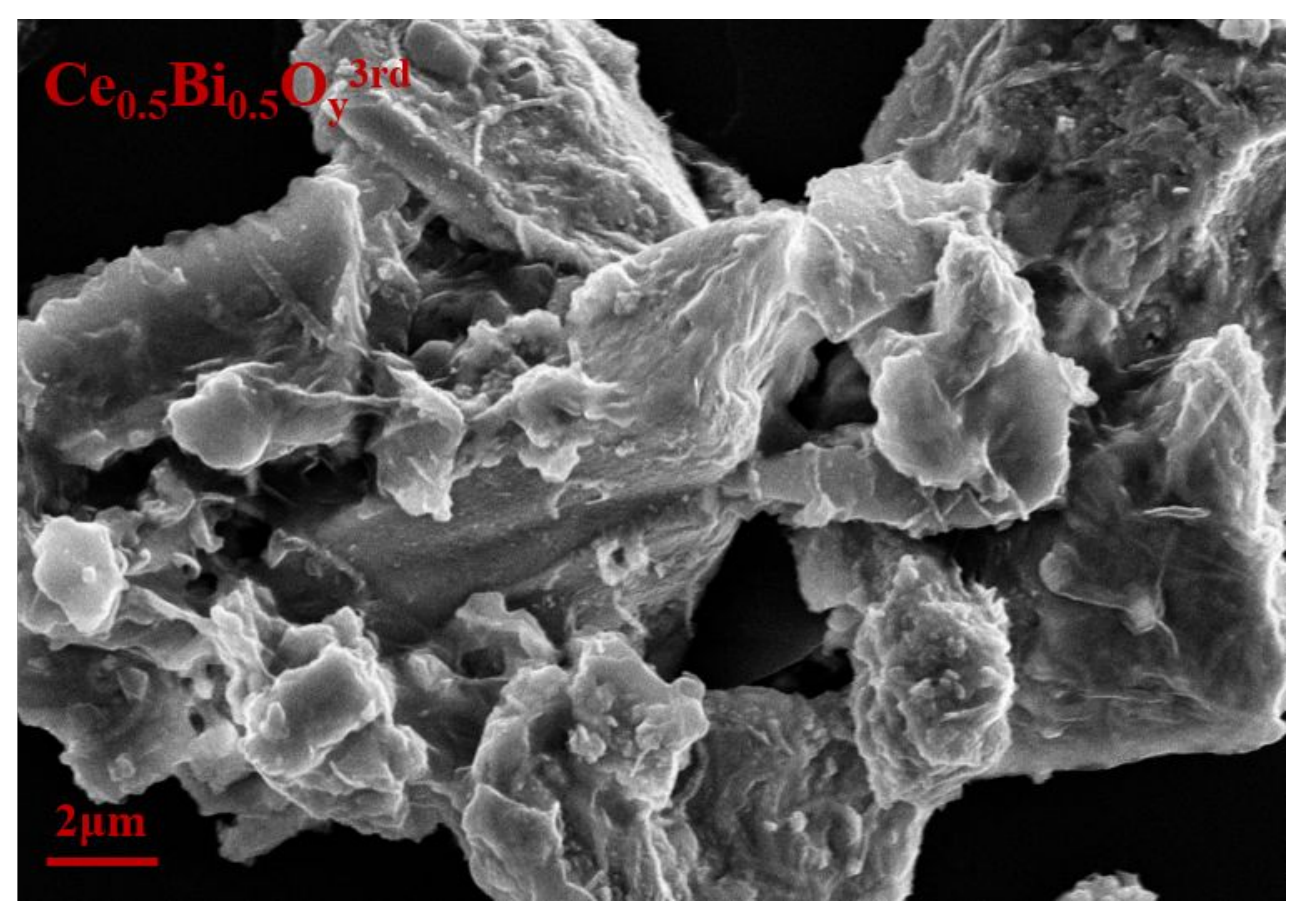

Fig. S3. SEM images of $\mathrm{Ce}_{0.5} \mathrm{Bi}_{0.5} \mathrm{O}_{\mathrm{y}}$ after the three cycles of testing at loose contact mode. 